\title{
BMJ Open Health workers' perspectives of a mobile health tool to improve diagnosis and management of paediatric acute respiratory illnesses in Uganda: a qualitative study
}

\author{
Laura Elizabeth Ellington (D) , ${ }^{1}$ Irene Najjingo, ${ }^{2}$ Margaret Rosenfeld, ${ }^{1,3}$ \\ James W Stout, ${ }^{1,4}$ Stephanie A Farquhar, ${ }^{4}$ Aditya Vashistha, ${ }^{5}$ Bridget Nekesa, ${ }^{2}$ \\ Zaituni Namiya, ${ }^{2}$ Agatha J Kruse, ${ }^{6}$ Richard Anderson, ${ }^{6}$ Rebecca Nantanda ${ }^{2}$
}

To cite: Ellington LE, Naijingo I, Rosenfeld M, et al. Health workers' perspectives of a mobile health tool to improve diagnosis and management of paediatric acute respiratory illnesses in Uganda: a qualitative study. BMJ Open 2021;11:e049708. doi:10.1136/ bmjopen-2021-049708

- Prepublication history and additional supplemental material for this paper are available online. To view these files, please visit the journal online. To view these files, please visit the journal online (http://dx.doi. org/10.1136/bmjopen-2021049708).

Received 02 February 2021 Accepted 02 July 2021

Check for updates

(C) Author(s) (or their employer(s)) 2021. Re-use permitted under CC BY-NC. No commercial re-use. See rights and permissions. Published by BMJ.

For numbered affiliations see end of article.

Correspondence to Dr Laura Elizabeth Ellington; lelling@uw.edu

\section{ABSTRACT}

Objectives Mobile health tools have potential to improve the diagnosis and management of acute lower respiratory illnesses (ALRI), a leading cause of paediatric mortality worldwide. The objectives were to evaluate health workers' perceptions of acceptability, usability and feasibility of Acute Lower Respiratory Illness Treatment and Evaluation (ALRITE), a novel mobile health tool to help frontline health workers diagnose, treat and provide education about ALRI in children $<5$ years.

Design A qualitative study including semistructured interviews with health facility administrators and focus groups with primary care health workers.

Setting Two federally funded Ugandan primary care health facilities, one peri-urban and one rural.

Participants We enrolled 3 health administrators and 28 health workers (clinical officers and nurses).

Intervention The ALRITE smartphone application was developed to help frontline health workers adhere to ALRI guidelines and differentiate wheezing illnesses from pneumonia in children under 5 years of age. ALRITE contains a simple decision tree, a partially automated respiratory rate counter, educational videos and an adapted respiratory assessment score to determine bronchodilator responsiveness. We performed a demonstration of ALRITE for participants at the beginning of interviews and focus groups. No participant had used ALRITE prior.

Results Themes impacting the potential implementation of ALRITE were organised using individual-level, cliniclevel and health-system level determinants. Individuallevel determinants were acceptability and perceived benefit, usability, provider needs and provider-patient relationship. Clinic-level determinants were limited resources and integration within the health centre. Systems-level determinants included medication shortages and stakeholder engagement.

Conclusions Incorporation of these themes will ready ALRITE for field testing. Early engagement of end users provides insights critical to the development of tailored mHealth decision support tools.

\section{Strengths and limitations of this study}

- By using a technology probe and human-centred participatory approach early in mHealth development, we engaged participants and gathered information not only about the Acute Lower Respiratory IIIness Treatment and Evaluation (ALRITE) tool but also contextual factors that are pivotal to the ultimate success of an mHealth application in this setting.

- We partnered with local health officials in the planning phase to encourage health worker attendance to information sessions, which translated to enrolling almost all eligible health workers, thus providing a more accurate and complete assessment at each study site.

- This study was limited by perspectives at two health centres, which may not reflect regional differences in resource availability, staffing and health workers' perceptions.

- Health worker perceptions were obtained without experience using ALRITE in clinical practice, which will be a focus of future work.

- We acknowledge that key team members who participated in all aspects of this project are American physicians/researchers who bring a different set of experiences and lens to this work, which may have influenced participants' responses and interpretation, but American team members worked in close partnership with Ugandan team members to ensure shared decision making and engagement with study participants.

\section{INTRODUCTION}

Acute lower respiratory illnesses (ALRI) remain a leading cause of mortality in children under 5 years, responsible for $15 \%$ of all deaths in this age range. ${ }^{2}$ Over 800 000 young children worldwide die of ALRI each year; 500000 of these deaths occur in 
sub-Saharan Africa. ${ }^{1-5}$ In Uganda, ALRI are responsible for $11 \%$ deaths in children under 5 years. ${ }^{56}$ ALRI encompass multiple disease processes that include bacterial pneumonia, viral pneumonia and wheezing illnesses. Differentiating between these diseases and choosing the appropriate treatment plan is challenging, especially where skilled personnel and diagnostic tools are lacking. The WHO Integrated Management of Childhood Illnesses (IMCI) provides guidelines for ALRI diagnosis and management, with emphasis on pneumonia and treatment with antibiotics. The IMCI was updated in 2014 to include assessment of wheezing and treatment with inhaled bronchodilators, ${ }^{7}$ but wheezing illness remains underdiagnosed and undertreated in low/middle-income countries (LMICs)..$^{8}$

Mobile phone use recently surpassed two-thirds of the global population and over $70 \%$ in Uganda, ${ }^{10}$ offering opportunities for digital health tools to enhance adherence to guidelines and build capacity through clinician education. ${ }^{11-16}$ Importantly, small pilot studies of mHealth tools based on WHO IMCI ALRI guidelines demonstrated promising preliminary results but have not addressed wheezing illness. ${ }^{17-20}$ To promote responsible, sustainable and high impact mHealth interventions in LMICs, the WHO recently released digital health guidelines recommending high quality research in fields of decision support and education. ${ }^{21}$

We developed the Acute Lower Respiratory Illness Treatment and Evaluation (ALRITE) mHealth application as a decision support tool to aid frontline health workers to improve diagnosis and treatment of ALRI in children under 5 years of age, with a particular focus on distinguishing wheezing illness from pneumonia. In order to address potential challenges with widespread ALRITE use, this study sought to understand determinants of successful ALRITE implementation from the end users' perspective. The objective of this study was to evaluate health workers' perceptions of feasibility, usability and acceptability of the ALRITE mHealth tool in two Ugandan primary care health centres. This user-centred, formative approach will inform further development of a locally relevant decision support tool to improve the diagnosis and treatment of ALRI in Ugandan health centres.

\section{METHODS}

\section{Study design}

This study uses a human-centred, or participatory, approach to examine frontline health workers' perceptions of ALRITE and its impact on their workflows and patient care. We developed an initial prototype of ALRITE and used it as a technology probe to gather insights about its feasibility, usability and acceptability. Technology probes are defined as instruments to " [collect] information about the use and users of the technology in a real-world setting', improve the intervention's design by meeting the needs and wishes of the user, and field-test. ${ }^{22}$ We used an exploratory qualitative study design to allow for deeper exploration into feasibility, usability and acceptability for the purposes of (1) improving the mHealth tool, (2) identifying barriers/facilitators beyond the tool itself to inform feasibility and implementation strategies and (3) determining quantitative outcomes measures for future studies (qual to QUAN mixed methods approach). ${ }^{23}$ The research team determined that quantitative survey data would have been inadequate to answer our research questions due to lack of depth, opportunity to probe and concerns about social desirability bias.

We conducted in-depth semistructured interviews with health facility administrators to understand clinic context, availability of resources, challenges, day-to-day operations and feasibility of ALRITE from a systems standpoint (online supplemental material). We conducted focus groups with primary care health workers (clinical officers and nurses) to understand how participants respond to peer responses and the forces that may influence their thinking and behaviour around the app, how this would affect patient-provider interactions and their reactions towards technology. All health worker participants had time to practise using ALRITE with clinical scenarios (online supplemental material) before focus groups to give participants a better understanding of the app, its content and usability to better inform their focus group responses.

\section{Study sites}

Both study sites were federally funded Health Center IV in Jinja district, Uganda and offer free healthcare. The peri-urban site is located $15 \mathrm{~min}$ driving from the city centre of Jinja, the second largest city in Uganda, and the rural site is located 45-60 min driving from Jinja. Both sites have inpatient and outpatient facilities and an operating theatre for obstetrics and urgent surgical cases.

The healthcare delivery system in Uganda has six levels that build on the previous level: (1) Health Centre II provides basic outpatient care; (2) Health Centre III has maternity services; (3) Health Centre IV has primary care, basic inpatient facilities and emergency obstetric care; (4) District Hospitals have general surgery, dental services and diagnostic services (ie, chest radiography and laboratory); (5) Regional Referral Hospitals have specialised care; (6) National Referral Hospital has additional specialised and sub-specialised services.

The two specific Health Centre IV were selected for this study based on prior research indicating that (1) adherence to IMCI was low, (2) consultations were performed by health workers with limited training, (3) antibiotics were overprescribed and (4) inhaled bronchodilators for wheezing illness were not prescribed. ${ }^{9}$

\section{Participants}

We recruited at least one health administrator (HA) for semistructured interviews from each study site. HAs were clinicians (medical doctors or clinical officers) who serve a director role in leadership and staff supervision at an individual health centre, termed locally as "health facility 
in-charges'. Eligible participants for focus groups were health workers (clinical officers or nurses) who had been working at the study site for at least 6 months and were responsible for outpatient care of children. None had used ALRITE prior. Clinical officers complete a 3-year diploma course in clinical medicine. Nurses in these health centres primarily act as clinicians due to staff shortages and task shifting. Prior to data collection, research team members met with officials at the Jinja District Health Office for approval, plan for disseminating study information to participating study sites and scheduling days for recruitment and data collection. Information sessions were coordinated with help from HAs at each study site to maximise participation. All health workers were notified about the session dates 1 week in advance and were invited to attend the information session even if not scheduled to work that day. The study team employed in-person information sessions for recruitment using convenience sampling. Sample size was determined by the number of health workers who showed up the scheduled days of data collection with the goal of recruiting all eligible health workers at each study site. All participants provided a written informed consent in English for their participation.

\section{ALRITE mHealth tool}

Based on a previous mHealth tool, mPneumonia, ${ }^{17} 18$ the ALRITE mHealth application was developed for smartphones to help frontline health workers adhere to IMCI guidelines and differentiate wheezing illnesses from pneumonia in children under 5 years of age. ALRITE contains a simple decision tree, a partially automated respiratory rate counter, educational videos (brief clips providing examples of children in respiratory distress, taken from WHO IMCI training videos with permission) and an adapted respiratory assessment score to determine bronchodilator responsiveness (figure 1). The algorithm walks the user through basic demographics, IMCI danger signs, medical history, physical exam and bronchodilator assessment (if appropriate). The final diagnoses include severe pneumonia or very severe disease, pneumonia $+/-$, wheezing illness, and cough or cold \pm wheezing illness. The WHO classification does not include a separate diagnosis of 'wheezing illness' but rather includes the diagnosis and treatment of wheezing as additional recommendations for the diagnoses of 'pneumonia' and 'cough or cold'. ${ }^{7}$ We added the term 'wheezing illness' to ALRITE diagnoses to prompt health workers to provide bronchodilators and refer for further assessment as necessary. The app is $27 \mathrm{MB}$ and was downloaded on supplied Android smartphones for study use.

\section{Data collection and management}

Prior to data collection, research assistants were trained and pretested focus group/interview guides through simulations with the research team. Demographic information was collected first on paper forms, then transferred to REDCap (Research Electronic Data Capture). ${ }^{24}$ Unique identifiers were used for each participant. Focus groups and interviews were performed primarily in English over the course of 1 week using interview/focus group guides (online supplemental information). Prior to focus groups, all health worker participants were given time to practise using ALRITE by going through at least two clinical scenarios individually or in small groups of up to three people (online supplemental information), while members of the study team (LEE, IN, MR, SAF, $\mathrm{BN}, \mathrm{ZN}$ ) asked for specific feedback, answered questions about the app and took notes. We performed a demonstration of ALRITE for participants at the beginning of interviews and focus groups. Ugandan research assistants did provide clarifications and some probing in the local language; some participant responses were given in the local language and translated to English for the study notes. All interviews and focus groups were digitally recorded, deidentified and transcribed into English without identifiers by IN, who is fluent in English and the local language. Transcriptions were reviewed by IN and LEE for content and cultural accuracy. Members of the study team (LEE, IN, MR, SAF) took notes during focus groups and interviews to augment and clarify the transcribed notes. Hard copy data were securely transported to Makerere University Lung Institute (Kampala, Uganda) for secure storage. No personal data will be transferred from the primary institution in Kampala, Uganda.

\section{Study team}

This was an international collaboration, including experts in public health, pulmonology/asthma, information and communication technology for development, humancomputer interaction and community-based interventions. Research assistants $\mathrm{BN}$ and $\mathrm{ZN}$ from Uganda
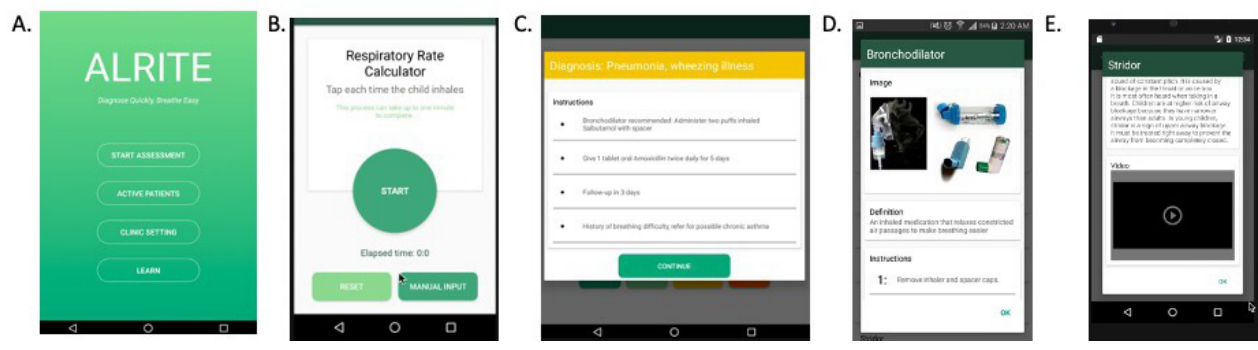

Figure 1 ALRITE sample screenshots. (A) Menu screen. (B) Respiratory rate counter. (C) Example of diagnosis and treatment recommendations. (D) Educational toolkit pop-up on bronchodilator administration. (E) Educational toolkit pop-up on stridor. 
experienced in qualitative interviewing and fluent in the local language led focus groups and interviews with the guidance of LEE, a content expert in the ALRITE app with previous qualitative experience in technology implementation. SAF provided qualitative expertise in design, data collection and analysis. RN provided local expertise in health systems, qualitative design and interview guides, and paediatrics. IN provided local research coordination and knowledge of the health system. Paediatric expertise was provided by RN and JWS with additional paediatric pulmonology expertise by MR and LEE. RA was instrumental in the design of mPneumonia and senior author on both manuscripts. ${ }^{17} 18$ ALRITE app design and development was performed by AJK, AV and RA, with additional expertise in usability testing and human-centred design by AV. We acknowledge that key team members who participated in all aspects of this project are American physicians and researchers who bring a different set of experiences and lens to this work, and that our positionality may have influenced participants' responses and interpretation. Working in partnership with our Ugandan team was critical to ensure shared decision making and our ability to work closely with the clinicians.

\section{Analysis}

We analysed our detailed notes and transcripts using a deductive thematic approach, whereby the researchers LEE, SAF and IN examined the data to identify common themes for each of the research questions based on a similar framework of a previously published mHealth technology. ${ }^{17} 18$ During the analysis, the team documented outlier or dissenting perspectives in order to provide a more complete picture of participant responses to ALRITE. First, LEE and SAF read through each of the transcripts and set of notes and then we developed a provisional framework based on primary research questions. LEE, SAF, MR, IN, BN and ZN held team meetings following each day of data collection to compile notes, review emerging themes and refine the coding framework. Codes were aggregated into major themes and subthemes by first annotating an online document of transcripts, then reorganising into a separate document, similar to but without the use of coding software. Additional meetings with the research team allowed for further refinement of themes and subthemes. Transcripts were re-read to ensure that preliminary results represented the majority of user feedback. The quotes were chosen to confirm and highlight themes and introduce diverging viewpoints not previously captured. The Standards for Reporting Qualitative Research were used to guide reporting. ${ }^{25}$

\section{Patient and public involvement}

While not involved in the initial design, conduct or reporting, study participants are involved in ultimate design and implementation of the intervention and are included in the dissemination plan, along with

\begin{tabular}{|c|c|c|}
\hline & Rural site & Peri-urban site \\
\hline In-depth interviews & $n=1$ & $n=2$ \\
\hline \multicolumn{3}{|l|}{ Role } \\
\hline Medical officer & 0 & 2 \\
\hline Clinical officer & 1 & 0 \\
\hline Male & 1 & 2 \\
\hline Focus groups & $n=12$ & $n=13$ \\
\hline \multicolumn{3}{|l|}{ Role } \\
\hline Clinical officer & 2 & 3 \\
\hline Nurse & 10 & 10 \\
\hline Female & 9 & 10 \\
\hline \multicolumn{3}{|l|}{ Age } \\
\hline$<30$ years & 5 & 5 \\
\hline $30-40$ years & 5 & 6 \\
\hline$>40$ years & 2 & 2 \\
\hline \multicolumn{3}{|c|}{ Experience in healthcare, years } \\
\hline$<5$ & 2 & 3 \\
\hline $5-10$ & 8 & 4 \\
\hline$>10$ & 2 & 6 \\
\hline
\end{tabular}

district-level and national-level health system members and patient caregivers.

\section{RESULTS}

\section{Participant and setting characteristics}

In January 2020, we enrolled 28 healthcare provider participants across two health centres in Uganda. Key stakeholders, including $3 \mathrm{HAs}$, took part in individual in-depth interviews, while 5 clinical officers and 20 nurses took part in three focus groups (table 1). Based on recommendations from the HAs from each site, we conducted separate focus groups for clinical officers $(n=3)$ and nurses $(n=10)$ at the peri-urban site to limit concerns around potential unequal power dynamics, but this was not deemed a concern at the rural site where one focus group was recommended. Interviews were approximately 30 min long, while focus groups were approximately 1.5 hours in length.

We identified several themes impacting the development and implementation of ALRITE in Ugandan health centres from the perspective of HAs and frontline health workers. Themes were organised by a social ecological model of determinants: individual, clinic and health system (figure 2). Individual-level determinants were acceptability and perceived benefit, usability, provider needs and provider-patient relationship. Clinic-level determinants were limited resources, integration within the health centre. Systems-level determinants included medication shortages and stakeholder engagement. Each theme is presented below in greater detail and with direct quotes that typify respondent comments. 


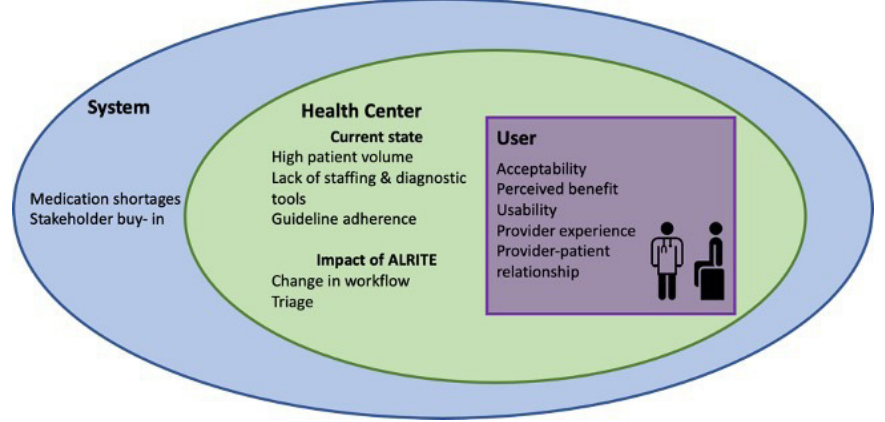

Figure 2 Frontline health workers' perspectives of determinants of ALRITE implementation.

\section{Individual-level}

Acceptability and perceived benefit of ALRITE

All health workers indicated they would like to have ALRITE available to use in their healthcare setting. Health workers also reported that they appreciated that ALRITE reminded them of important medical questions to ask and key components of the physical exam.

It helps us to remember the clear assessment of these children because at times you are rushing and forget to assess something. You go with what you see quickly, but the app gives you the procedure to follow. It also helps in giving the right doses. (HW-002-FG2)

They responded positively to the integrated respiratory rate counter. Health workers' eagerness to learn was apparent during focus groups and interviews. Indeed, many health workers reported the educational videos were one of their favourite features of ALRITE. In addition to data gathering, health workers liked the information management capabilities, including medication dosing, which is generally age-based or weight-based for children.

The part of the app that I like mainly are the videos. It is good because it helps diagnosing and guides through the right treatment hence saving patient time. (HA-001)

[The respiratory rate counter] is convenient because you may not have a watch. (HW-007-FG3)

It is important to acknowledge the novelty of ALRITE as a mHealth app technology likely contributed to high acceptability by health workers as well.

[Providers] usually like new technology, I think they will be excited to use it and therefore they are likely to download [the app]. In addition, people prefer digital information than opening and reading what is in the [IMCI] book. (HA-003)

Respondents had ideas for improving acceptability, appropriateness and potential benefit of ALRITE. Health workers asked for additional automated or semiautomated smartphone tools, such as pulse oximetry or digital auscultation to be integrated into ALRITE. Multiple health workers commented on the potential for storage of clinical information. One health worker wanted to use it as a personal quality control device to review his previous diagnoses and treatment plans. A few wanted the app expanded to other disease processes and age groups. Some health workers suggested incorporating additional educational components targeted to patients and families. One health worker suggested incorporating risk stratification for children with chronic disease and environmental risk factors (ie, smoke exposure, crowded housing) in order to focus on prevention.

[In the app], we are missing [a question on] the type of fuel used at home to cook and source of light. Some produce a lot of smoke. [By offering recommendations, families] can change the way of cooking, hence reducing exposures. This could help in prevention [of respiratory diseases]. We therefore can make a recommendation and follow-up in about 6 months. (HW-005-FG3)

\section{ALRITE usability}

We defined usability as 'the design factors that affect the user experience of operating the application's device and navigating the application for its intended purpose'. ${ }^{18}$ ALRITE features that contributed to a positive user experience included overall design, simplicity, flow and clarity of diagnosis. Generally, health workers thought the app was easy to follow and would be quick to get to diagnosis.

It saves time. You diagnose very fast and you are able to know the treatment to give so it improves on the appropriate management of patients. (HW-001-FG1

When given the opportunity to use the mHealth tool, health workers who owned smartphones were more facile with ALRITE than those who owned simple mobile phones. Importantly, after practising with ALRITE and receiving coaching from the research team, all health workers became more facile using ALRITE. Proficiency with the app was not formally tested.

Health workers provided valuable feedback to improve the usability of ALRITE, including minor changes to the visual display, layout and flow of the app. For example, health workers recommended larger font for better visualisation. They also recommended using a patient age group instead of date of birth for two reasons: (1) health workers had difficulty using the calendar function, and (2) caregivers may not know a child's date of birth, so asking for a child's age group is a standard practice.

\section{Provider-specific needs}

Another important consideration to ALRITE acceptability is the end user's experience with smartphones. In our study, all health workers owned a mobile phone, of which approximately $60 \%$ owned a smartphone. Most who owned a smartphone used social media or communication apps. No health workers we spoke with were using mHealth apps, and only a few had heard of these types of apps. 
All those with smartphones preferred that ALRITE be directly downloaded to their personal devices compared with clinic-supplied devices, as they would be more likely to use the app if it were readily available on their own smartphones. Additionally, if health workers used the app on their own smartphones rather than a clinicsupplied device, they said they would be less likely to lose or misplace the device. However, one health worker reminded the group that not everyone has a smartphone, so smartphones would need to be made available to individuals without a personal device. Health workers were pleased that the size of the app was only $(27 \mathrm{Mb})$.

The provider's training level was also an important factor. In different levels of health centres in Uganda, there are health workers with varying levels of training and provider roles, ranging from nurse to medical officer. End users with limited clinical training may be more likely to use ALRITE in practice to help with clinical decision making than others with more training or clinical experience who may not think additional clinical decision support adds value to their clinical care.

There are incidences when the doctors are not at the clinic and the nurse needs to make a diagnosis and give treatment as well. ALRITE will save time. (HA-001)

\section{Provider-patient relationship}

Health workers had some concerns regarding ALRITE use in their clinic. Some thought that using the app in front of patients and families would reduce the quality and quantity of personal interaction at a clinic visit. Some also expressed concern that if they used ALRITE to help make clinical decisions, families would lose trust in health workers' ability to diagnose and treat. However, one respondent also suggested that the app could be used as an educational tool for families and help build trust during the visit.

The first challenge is on the side of our clients. When you are busy using the app, the client might think you're neglecting her or him and you're busy on WhatsApp, and secondly, a client might think you're not knowledgeable enough since you're using a phone and lose trust in you thinking you don't know what to do... But I think I can start by engaging the patients and informing them that what I am going to do is for your good, I am not just looking for answers but rather improving diagnosis for your child. (HW-003-FG2)

\section{Clinic-level}

Limited resources

\section{High patient volume and limited staffing}

At the peri-urban site, 100-300 patients are seen in the ambulatory clinic daily, $60 \%$ of which are children (IC-001). Similarly, at the rural site, a stakeholder reported, 'we see about 100-200 patients daily... and about $45 \%$ of these are children less than 5 years' (IC-003). Stakeholders identified the most common paediatric conditions: malaria, ALRI and diarrhoea. A combination of clinical officers and nurses without specialty paediatric training see paediatric patients at both sites. The periurban site also staffs a few general medical doctors, but they are not always on site. All health workers reported they received IMCI training, from which Uganda Clinical Guidelines are derived. ${ }^{26}$ Health workers reported that visit length typically ranges from 10 to $15 \mathrm{~min}$, but a few reported they often take less than $10 \mathrm{~min}$.

In assessing children, we have a challenge with patient load with few trained health workers who can assess patients. It's a facility in a semi-urban area so the numbers are big with few health workers, and treatment is not always available. (HA-001)

\section{Limited resources for the diagnosis of paediatric respiratory disease}

To diagnose respiratory disease, both sites reported use of stethoscopes, although these are not universally available, nor are they required to use IMCI. They rely on personal watches to count respiratory rate, but not everyone has a watch. Pulse oximetry is not typically available.

We have one pulse oximeter in [the operating] theater, but we are currently not using it because it gives confusing results. (HA-003)

It is not a problem [to use a stethoscope], but if it is not available, we resort to the IMCI approach where you depend on a physical exam [without a stethoscope]. In addition, what compromises quality is the number of patients waiting in the line to be reviewed, and you may end up missing out on an important indicator. (HW-002-FG3)

\section{Limited resources and training affecting adherence to WHO $\mathrm{MCl}$}

All health workers received WHO IMCI training. However, they reported that adherence to IMCI can be challenging for a number of reasons. First, IMCI incorporates respiratory rate and evaluation of respiratory distress into its clinical decision algorithm. Some health workers reported that counting respiratory rate is impossible without a watch or timer. Second, IMCI recommends evaluation of wheezing, but this exam finding is challenging to diagnose, especially without a stethoscope.

[Chest] indrawing is easier [to assess] compared to wheezing. (HW-001-FG3)

Monitoring during the care of these children or reassessing the vitals is a challenge. (HW-003-FG3)

Health workers frequently denied opportunities for robust continuing medical education or refresher trainings for IMCI.

We have sent people for IMCI training. We also have a national trainer at the facility who organizes [continuous medical education], but the turnout of health 
workers is usually not good. Usually, when people do something for many years, they tend to think there is nothing new they can learn. (HA-003)

Third, availability and use of the IMCI materials are limited in health centres. IMCI can be available in paper or electronic form. However, health workers reported that the paper form is not convenient to use due to the size of the booklet and the likelihood of misplacing it. One HA reported that his health centre had IMCI installed on a laptop, but the laptop broke and was not replaced.

\section{ALRITE integration into existing health system Change to workflow}

Integrating ALRITE into clinical practice will inevitably change clinic workflow. It may contribute to duplicative work because the current system includes entering data into a written health record. Interestingly, this was not brought up as a concern during focus groups or interviews. Furthermore, using a new technology will be slow at first and may make patient encounters longer rather than shorter in an already busy clinic. Health workers reported that they would need to practise with ALRITE prior to using it with patients to improve work efficiency.

At first, it's likely to slow the work because we may be learning the app but with time it will become part of us, and we become part of it so it will ease the work... we need to be familiar with it to help us save time so that patients do not see us take a lot of time on the phones. (HW-002-FG2)

I think we shall have to sort out those with respiratory illnesses at triage which is different from what is being done currently where all patients follow the same assessment route regardless of condition. (HW-001-FG1)

Furthermore, current practice in Ugandan health centres does not routinely include reassessment of patients after a bronchodilator trial, which is necessary to ascertain whether patients would benefit from treatment with a bronchodilator. Most health workers thought it would be feasible to reassess patients if warranted; however, a few health workers reported that many patients leave after the initial assessment. Lack of reassessment would limit providers' ability to determine bronchodilator responsiveness, an important factor in diagnosing wheezing illnesses and asthma in young children and therefore an important component of ALRITE.

We reassess only those who are admitted on the wards. We reassess if the child worsens, but if they are improving, we reassess them the next day during the ward rounds. I think it's important to integrate the app into the system because it gives reminders about reassessing a patient. (HA-001)

There is no opportunity [to reassess children] because most of them come from far and do not usually come back [after treatment is prescribed]. (HW-001-FG3)
Triage

One current challenge and potential opportunity for ALRITE integration is in patient triage. HAs reported no formal triage process to risk-stratify patients as they present to care. There is also no separate paediatric clinic. All patients are seen in the order they arrive, whether adults or children. One HA saw ALRITE implementation as an opportunity to establish triage at their health centre. He suggested that ALRITE could be used earlier when paediatric patients arrive to the clinic to prioritise those with WHO danger signs and acute respiratory distress.

[Challenges include] lack of a dedicated clinician to manage children and lack of enough consultation rooms. There is also a knowledge gap in assessing children. We do not have a triage area where we are able to prioritize those with worse conditions. We usually just do visual observation of who is an emergency situation instead of taking medical history and a few vitals. The other issue is we don't differentiate children from adults, they all go through the same entry point... The other [issue] is lack of an emergency unit for children with severe difficulty in breathing. (HA-003)

\section{Systems-level}

Medication shortages impacting ALRITE management

Aside from features of ALRITE itself, we explored other factors that would impact feasibility of ALRITE at a systems level. ALRITE recommends treatment for children with respiratory illnesses, including antibiotics for pneumonia and inhaled bronchodilators with/without systemic corticosteroids for wheezing. Oral salbutamol is generally available at the health centres and sometimes used for children but carries a high side effect profile and is not recommended for acute wheezing in children. ${ }^{27}{ }^{28} \mathrm{HA}$ at both sites reported very limited availability of inhaled bronchodilators (2-3 inhalers every 2 months). Health workers may prescribe medications if not available on site, but this requires family members to pay out-of-pocket for prescribed medications at an off-site pharmacy or higherlevel health centre. Even if ALRITE improves diagnosis of wheezing illness, its impact and feasibility will be greatly limited if appropriate treatment is not readily available.

We are not independent when it comes to drugs. Supplies are from National Medical Stores, and they usually give what they have unless you have an independent source outside of the usual supply chain. (HA-002)

The app talks about the bronchodilator, but it doesn't talk about other drugs to give. Here at the low-level facilities we do not have the bronchodilators. (HW-002-FG1)

\section{Stakeholder buy-in}

While not a common theme, one HA emphasised the importance of engaging stakeholders early for successful 
implementation. Specifically, the administrator explained that the Ministry of Health in Uganda and local district health officials would need to approve the app prior to large scale distribution across public and private facilities. Additionally, support at these leadership levels will be critical for widespread uptake and implementation of ALRITE.

\section{DISCUSSION}

In this study, we identified key determinants towards successful implementation of ALRITE, our mHealth decision support tool, from frontline health workers' perspectives (figure 2). In addition to ALRITE-specific determinants, health workers and administrators identified important individual-level, clinic-level and health systems-level determinants and offered innovative ideas for future app development. Overall, these results support ongoing development of ALRITE for potential integration into routine clinical care and underscore the importance of user-centred design early in development prior to implementation of a new technology. ALRITE, if successfully implemented, has the potential to improve childhood morbidity and mortality in three major ways: (1) increased awareness, diagnosis and treatment of wheezing illness, (2) improved IMCI guideline adherence through prompts and education and (3) effective triage of critically ill infants and children. Potential challenges identified include changes to the provider-patient relationship, time constraints and medication shortages. However, through thoughtful design and implementation, ALRITE has potential to overcome these challenges by enhancing the provider-patient relationship through education and improved management, improving clinical efficiency through a streamlined process and increasing supply of life-saving medications such as inhaled bronchodilators through increased awareness, advocacy and demand.

Additional strengths of the study include using a technology probe and human-centred, participatory approach early in mHealth development to engage participants and gather information not only about the specific mHealth tool but also to build an underpinning knowledge of factors that are pivotal to the ultimate success of a mHealth application. We partnered with local health officials in the planning phase to encourage health worker attendance to information sessions, which translated in almost all eligible health workers at each site participating in the study to provide a more accurate and complete on-the-ground assessment at each study site. We also included HAs as participants to provide a broader understanding of the clinic context, challenges, day-to-day operations and feasibility of ALRITE from a systems standpoint, adding a unique perspective to the health workers' responses.

Previous studies have also evaluated mHealth decision support based on WHO IMCI. ${ }^{17-19}$ The predecessor to ALRITE, mPneumonia, demonstrated high acceptability and usability in pilot studies in Ghana. ${ }^{17}{ }^{18}$ Unlike ALRITE, mPneumonia was designed to use on clinicsupplied tablets. Health workers were not as familiar with smartphones and had difficulty navigating the application and general tablet use. ${ }^{17}$ Furthermore, health workers expressed potential challenges of mPneumonia including access to electricity and added time to patient encounters. ${ }^{18}$ The disparate results between mPneumonia and ALRITE likely reflects interval improvements in devices and software as well as additional experience with smartphones given growing mobile phone use worldwide.

Two important systematic reviews of health workers' perspectives using mHealth in primary care highlighted similar themes. ${ }^{21} 29$ Decision support mHealth tools achieved high acceptability, with health workers reporting increased efficiency, better access to information and improved adherence to guidelines. ${ }^{21}$ Similar to our results, some health workers were concerned mHealth may negatively impact the provider-patient relationship, ${ }^{21}$ but this concern has not been evaluated by patients or caregivers. Contrary to our results, some health workers were concerned the algorithm was too prescriptive for clinical decision making. ${ }^{21}$ We found that health workers appreciated the simple ALRITE algorithm, which may reflect the limited clinical training in our study population. Additional factors influencing health workers' acceptability of mHealth technologies were cost to the health worker, previous mobile phone experience and increased time/ workload. ${ }^{29}$ While our study did not evaluate cost, as ALRITE would be a free application, health workers did appreciate that ALRITE had a small footprint (27 Mb) so would not require much data or take up much smartphone memory. Conversely, health worker perceptions of ALRITE did not change based on prior mobile phone experience, but those with smartphone experience were much more facile with the app.

There has been a recent explosion of digital health tools for use in LMICs, but evidence on effectiveness and scale-up has been lacking. ${ }^{11} 163031$ An early humancentred approach to evaluation is critical to better understand determinants of successful implementation and to guide further mHealth design. Therefore, we included HAs and frontline health workers early in the development of ALRITE as participants to better inform acceptability, appropriateness and feasibility of its use in Ugandan health centres. Through stakeholder interviews and health worker focus groups, we not only received important feedback to improve ALRITE, but also gained a richer understanding of the health setting and potential systems-based and individual level challenges to implementation.

This study had important limitations. First, perceptions of health workers were limited to two health facilities in Uganda. We purposefully chose one peri-urban and one rural health centre to better understand differences in resource availability, staffing and health workers' perceptions. However, there may be additional regional differences in perceptions of and comfort with ALRITE that 
have yet to be explored. Second, it may be possible that we did not capture the full breadth of perspectives, as health workers with dissenting opinions may not have felt comfortable speaking up during focus groups. We tried to address this by probing for dissenting opinions during focus groups and while health workers were practising with the app in smaller groups. Third, we did not perform formal quantitative usability evaluations. A formal evaluation of end user proficiency was not the objective of this study because the ALRITE app was still in the prototype phase. Finally, health worker's perceptions were obtained without experience using ALRITE in clinical practice. This understanding of feasibility in clinical care will be a major focus of future work.

Next steps include updating ALRITE based on user feedback and field testing with frontline health workers. We will also address important potential barriers for implementation, including engaging caregivers, streamlining the ALRITE app to limit any negative effect on existing workflow, developing training programmes, ensuring readily available technical support and engaging key stakeholders at the Uganda Ministry of Health and district health leadership to support further research, medication supply and ultimate implementation of ALRITE.

\section{CONCLUSION}

Taken together, these results provide a detailed, on-theground assessment of the opportunities and challenges in the respiratory assessment, diagnosis and treatment of ALRI in young children. Further, the engagement of health workers and richness of data collected support the use of human-centred approaches early-on to identify factors that are pivotal to success of a mHealth application. Finally, our results support the continued development of tailored mHealth tools for decision support in LMICs based on high user acceptability and usability.

\section{Author affiliations}

${ }^{1}$ Pediatrics, University of Washington School of Medicine, Seattle, Washington, USA ${ }^{2}$ Makerere University Lung Institute, Makerere University College of Health Sciences, Kampala, Uganda

${ }^{3}$ Department of Epidemiology, University of Washington School of Public Health, Seattle, Washington, USA

${ }^{4}$ Department of Health Systems and Population Health, University of Washington

School of Public Health, Seattle, Washington, USA

${ }^{5}$ Computer Science, Cornell University, Ithaca, New York, USA

${ }^{6}$ Computer Science and Engineering, University of Washington, Seattle, Washington, USA

Acknowledgements We would like to sincerely thank all health workers and health facility in-charges who participated in this study. We are also grateful to the Jinja District Health Office who gave permission and support to conduct the study.

Contributors LEE, MR, JWS, AV, RA and RN contributed to the concept and study design. AJK developed the mobile health application with mentorship from AV and RA. LEE, IN and RN coordinated and supervised data collection from the sites. LEE, MR, SAF, IN, BN and ZN performed data collection. LEE, SAF and IN analysed the data, and all authors contributed to interpretation. LEE wrote the draft of the manuscript. RN provided oversight of the project. All authors worked collaboratively to review, edit and approve the final manuscript.

Funding This work was supported by the University of Washington Global Innovation Fund, the Firland Foundation, the Arthur Rosenfeld Endowment for Paediatric Pulmonary Fellows, and the NIH NHLBI (Grant number 5K12HL137940).

Competing interests None declared.

Patient consent for publication Not required.

Ethics approval The study was approved by the Mulago Hospital Research and Ethics Committee and Uganda National Council for Science and Technology (HS2692). The study was reviewed and received exempt status from the University of Washington (STUDY0007895). Written informed consent was obtained from all participants in accordance with international and local regulations.

Provenance and peer review Not commissioned; externally peer reviewed.

Data availability statement Data are available upon reasonable request. All data relevant to the study are included in the article or uploaded as supplementary information. Data supporting the findings are available within the manuscript. Additional quotes are available upon reasonable request to the corresponding author.

Supplemental material This content has been supplied by the author(s). It has not been vetted by BMJ Publishing Group Limited (BMJ) and may not have been peer-reviewed. Any opinions or recommendations discussed are solely those of the author(s) and are not endorsed by BMJ. BMJ disclaims all liability and responsibility arising from any reliance placed on the content. Where the content includes any translated material, BMJ does not warrant the accuracy and reliability of the translations (including but not limited to local regulations, clinical guidelines, terminology, drug names and drug dosages), and is not responsible for any error and/or omissions arising from translation and adaptation or otherwise.

Open access This is an open access article distributed in accordance with the Creative Commons Attribution Non Commercial (CC BY-NC 4.0) license, which permits others to distribute, remix, adapt, build upon this work non-commercially, and license their derivative works on different terms, provided the original work is properly cited, appropriate credit is given, any changes made indicated, and the use is non-commercial. See: http://creativecommons.org/licenses/by-nc/4.0/.

ORCID iD

Laura Elizabeth Ellington http://orcid.org/0000-0001-7904-4249

\section{REFERENCES}

1 Liu L, Oza S, Hogan D, et al. Global, regional, and national causes of child mortality in 2000-13, with projections to inform post-2015 priorities: an updated systematic analysis. Lancet 2015;385:430-40.

2 GBD 2015 Child Mortality Collaborators. Global, regional, National, and selected subnational levels of stillbirths, neonatal, infant, and under-5 mortality, 1980-2015: a systematic analysis for the global burden of disease study 2015. Lancet 2016;388:1725-74.

3 Walker CLF, Rudan I, Liu L, et al. Global burden of childhood pneumonia and diarrhoea. Lancet 2013;381:1405-16.

4 Zar HJ, Ferkol TW. The global burden of respiratory disease-impact on child health. Pediatr Pulmonol 2014;49:430-4.

5 McAllister DA, Liu L, Shi T, et al. Global, regional, and national estimates of pneumonia morbidity and mortality in children younger than 5 years between 2000 and 2015: a systematic analysis. Lancet Glob Health 2019;7:e47-57.

6 IHME. Uganda country profile 2016, 2016. Available: http://www. healthdata.org/uganda [Accessed 19 Oct 2018].

7 World Health Organization. Integrated management of childhood illness, 2014.

8 Nantanda R, Tumwine JK, Ndeezi G, et al. Asthma and pneumonia among children less than five years with acute respiratory symptoms in Mulago Hospital, Uganda: evidence of under-diagnosis of asthma. PLoS One 2013;8:e81562.

9 Kjærgaard J, Anastasaki M, Stubbe Østergaard M, et al. Diagnosis and treatment of acute respiratory illness in children under five in primary care in low-, middle-, and high-income countries: a descriptive fresh air study. PLoS One 2019;14:e0221389.

10 (CIPESA) TCollPfEaSA. National information technology survey 2017/18 report. CIPESA, 2018: 274.

11 Agarwal S, Perry HB, Long L-A, et al. Evidence on feasibility and effective use of mHealth strategies by frontline health workers 
in developing countries: systematic review. Trop Med Int Health 2015;20:1003-14.

12 Källander K, Tibenderana JK, Akpogheneta OJ, et al. Mobile health (mHealth) approaches and lessons for increased performance and retention of community health workers in low- and middle-income countries: a review. J Med Internet Res 2013;15:e17.

13 Lodhia V, Karanja S, Lees S, et al. Acceptability, usability, and views on deployment of peek, a mobile phone mHealth intervention for eye care in Kenya: qualitative study. JMIR Mhealth Uhealth 2016;4:e30.

14 Medhanyie AA, Little A, Yebyo $\mathrm{H}$, et al. Health workers' experiences, barriers, preferences and motivating factors in using mHealth forms in Ethiopia. Hum Resour Health 2015;13:2.

15 Vélez O, Okyere PB, Kanter AS, et al. A usability study of a mobile health application for rural Ghanaian midwives. J Midwifery Womens Health 2014;59:184-91.

16 Aranda-Jan CB, Mohutsiwa-Dibe N, Loukanova S. Systematic review on what works, what does not work and why of implementation of mobile health (mHealth) projects in Africa. BMC Public Health 2014;14:188.

17 Ginsburg AS, Delarosa J, Brunette W, et al. mPneumonia: development of an innovative mHealth application for diagnosing and treating childhood pneumonia and other childhood illnesses in low-resource settings. PLoS One 2015;10:e0139625.

18 Ginsburg AS, Tawiah Agyemang C, Ambler G, et al. mPneumonia, an innovation for diagnosing and treating childhood pneumonia in low-resource settings: a feasibility, usability and acceptability study in Ghana. PLoS One 2016;11:e0165201.

19 Rambaud-Althaus C, Shao A, Samaka J, et al. Performance of health workers using an electronic algorithm for the management of childhood illness in Tanzania: a pilot implementation study. Am J Trop Med Hyg 2017;96:249-57.

20 Keitel K, D'Acremont V. Electronic clinical decision algorithms for the integrated primary care management of febrile children in low-resource settings: review of existing tools. Clin Microbiol Infect 2018;24:845-55

21 World Health Organization. WHO guideline: recommendations on digital interventions for health system strengthening. Geneva, 2019.

22 Hutchinson $\mathrm{H}$, Mackay W, Westerlund B. Technology probes: inspiring design for and with families. Proceedings of the SIGCHI Conference on Human Factors in Computing Systems, Ft. Lauderdale, Florida, USA, 2003:17-24.

23 Creswell JW, Plano Clark VL. Designing and conducting mixed methods research. Third edition. Thousand Oaks, California: SAGE, 2018.

24 Harris PA, Taylor R, Thielke R, et al. Research electronic data capture (REDCap)--a metadata-driven methodology and workflow process for providing translational research informatics support. J Biomed Inform 2009;42:377-81.

25 O'Brien BC, Harris IB, Beckman TJ, et al. Standards for reporting qualitative research: a synthesis of recommendations. Acad Med 2014;89:1245-51.

26 Ministry of Health Uganda. Uganda clinical guidelines. Fourth ed. Kampala, Uganda: Ministry of Health Uganda, 2016.

27 MCR VISION, Inc. Global strategy for asthma management and prevention. MCR VISION, Inc, 2006.

28 World Health Organization. World Health organization model list of essential medicines for children. 7th Edition. Geneva: World Health Organization, 2019.

29 Odendaal WA, Anstey Watkins J, Leon N, et al. Health workers' perceptions and experiences of using mHealth technologies to deliver primary healthcare services: a qualitative evidence synthesis. Cochrane Database Syst Rev 2020;3:CD011942.

30 Tomlinson M, Rotheram-Borus MJ, Swartz L, et al. Scaling up mHealth: where is the evidence? PLoS Med 2013;10:e1001382.

31 Hall CS, Fottrell E, Wilkinson S, et al. Assessing the impact of mHealth interventions in low- and middle-income countries--what has been shown to work? Glob Health Action 2014;7:25606. 